Beyond the dyad:

A review of the novel insights gained from studying consensual non-monogamy

\author{
Rhonda N. Balzarini ${ }^{1}$ \\ Amy Muise ${ }^{2}$ \\ ${ }^{1}$ Department of Psychology, Texas State University, San Marcos, TX, United States \\ ${ }^{2}$ Department of Psychology, York University, Toronto, ON, Canada
}

This is the accepted, pre-publication version of the article, which appears in:

Balzarini, R. N. \& Muise, A. (2020). Beyond the dyad: A review of the novel insights gained from studying consensual non-monogamy. Current Sexual Health Reports, in press.

Correspondence concerning this article should be addressed to Rhonda Balzarini, Department of Psychology, Texas State University, San Marcos, TX 78666. E-mail: rnb85@txstate.edu or balzarinir@gmail.com. 


\begin{abstract}
Purpose of review: The purpose of this review is to highlight the major advancements in our understanding of consensually non-monogamous (CNM) relationships_or intimate relationships between three or more people who are non-exclusive sexually and/or emotionally. We aim to review key insights from research on the benefits (i.e., diversification of need fulfillment) and challenges (i.e., continued stigma) of CNM relationships and how research on CNM highlights some limitations of the existing theories of relationships.
\end{abstract}

Recent findings: The last two decades have seen a trend towards increasing diversity of family structures. Although monogamy remains the most common relationship structure, CNM relationships are increasing in prevalence and in interest to both lay people and researchers. Recent research has begun to uncover novel insights into who is more likely to be drawn to and engage in CNM, how CNM relationships compare to monogamous relationships, and the potential benefits and challenges of engaging in CNM relationships.

Summary: While people in CNM relationships still experience stigma, for those who desire such relationships, CNM can be a viable and healthy alternative to monogamy, and may even help people meet more of their needs through diversifying need fulfilment across multiple partners. Despite this, many existing relationships theories are not inclusive of CNM relationship experiences and aspects of existing theories of positive relationships and sexuality may not extend to people in CNM relationships. Moving forward it is important to consider whether our concepts and measures are inclusive to people in diverse relationships, including those in CNM relationships.

Keywords: consensual non-monogamy; romantic relationships; sexuality; need fulfillment; relationship quality; stigma 


\section{Beyond the dyad: A review of the novel insights gained from studying consensual \\ non- monogamy}

The last two decades have seen rapid changes in Western families, with a trend towards increasing diversity of family structures. Longer life spans (1), along with increases in interracial, inter-religious, and same-sex marriages (2), and steep fertility declines (3-4) are only a few of the social trends that are increasing the diversity of families and relationships.

However, the nuclear monogamous family remains a powerful normative ideal in much of the Western world (5-6), and people who do not follow this pattern may be considered deviant or not even families at all (7-8). While monogamy remains the most common romantic relationship arrangement in most parts of the world, consensual non-monogamy $(\mathrm{CNM})$ — or intimate relationships between three or more people who are non-exclusive sexually and/or emotionally (9-10) - is increasingly prevalent and becoming more visible in mainstream media and in societies as well (11). In fact, public interest in CNM relationships has increased dramatically in recent years, with searches for information on polyamorous relationships, one type of CNM relationship, being listed among the top 10 relationship queries by Google in 2017 (12). Increased interest in CNM is not only apparent in rising Google searches (12; also see 13), but in heightened media attention, with shows like "You Me and Her" and "Unicornland," as well as the inclusion of polyamory as a relationship orientation on the popular dating website OKCupid (14), and in more researchers examining the prevalence and outcomes of CNM arrangements $(11 ; 15)$.

Over the past decade research on CNM relationships has gained a greater presence in relationship and sexuality research (16) and has begun to uncover novel insights into who is more likely to be drawn to and engage in CNM, how CNM relationships compare to 
monogamous relationships, and the potential detriments (e.g., stigma) and benefits (e.g., diversified need fulfilment) of engaging in CNM relationships. This research suggests that for those who desire such relationships, CNN can be a viable and healthy alternative to monogamy (e.g., it can be just as satisfying as a monogamous relationship; $15 ; 17$ ), and having multiple simultaneous relationships can help people meet more of their fundamental needs (e.g., 18-20). The purpose of this review is to highlight the major advancements in our understanding of CNM relationships based on the most recent work in the field, discuss the ways in which insights from research on CNM relationships raise questions about the inclusivity of relationship theories (e.g., commitment being equated with monogamy or exclusivity), and challenge the boundary conditions of existing theories (e.g., attachment theory; 21). Through this review and identification of novel advancements in the field, we provide a summary of the current research on CNM relationships and discuss directions for future work.

\section{Prevalence and Engagement in CNM}

Past research suggests that a non-trivial number of people have engaged in or are interested in CNM relationships. For example, research using nationally representative samples of people from the United States and Canada have shown that approximately 4-6\% of individuals report currently being in a CNM relationship (22-23). And, far more people have experience with or interest in CNM, with more than $20 \%$ of Americans indicating previous participation in some form of CNM in their lifetime (23) and with CNM sexual experiences (e.g., a threesome) emerging as the most common sexual fantasy people report (24). Furthermore, the prevalence of experience with CNM is even higher among people who identify as bisexual, with approximately $33 \%$ reporting previous participation in CNM (23). 
Importantly, $\mathrm{CNM}$ is an umbrella term for various non-monogamous relationship agreements that differ based on the degree to which sexual and emotional needs are consensually fulfilled outside of a romantic dyad (16). The three types of CNM relationships that are often delineated are swinging, open, and polyamorous relationships $(15 ; 25 ; 26)$. Swinging relationships involve temporarily swapping or exchanging partners among couples, often in the context of a specific social event (26-27). Open relationships involve extradyadic sex without love and without a romantic partner's participation $(16 ; 26 ; 28 ; 29)$. Polyamorous relationships permit loving more than one person, and typically consist of multiple, emotionally close relationships $(16 ; 26)$. Although CNM affords emotional and sexual connections with multiple partners simultaneously, polyamory tends to involve close emotional with additional partners than swinging and open relationships, which tend to be primarily about sexual openness $(5 ; 15 ; 30 ; 31)$.

People in CNM relationships often have their own unique agreements with partners and CNM relationship can take on many different structures, but one of the most common configurations, at least for people in polyamorous relationships, are a primary-secondary relationship configuration $(17 ; 32)$. In this configuration, a primary relationship is between two partners who typically share household finances, live together, and who are married, and relationships with partners beyond the primary relationship are often referred to as secondary, non-primary, or tertiary partners $(17 ; 27 ; 33)$. Although less research has examined the configurations of people in open and swinging relationships, because these relationships primarily involve extradyadic sexual encounters, they often follow a similar structure with one partner serving as a primary partner and the other(s) as non-primary (e.g., 34; 35).

\section{Challenges of Deviating Beyond the Dyad: Stigma Towards CNM Relationships}


Despite the growing prevalence, people in CNM relationships are stigmatized (36). For example, 26-43\% of people in polyamorous relationships report experiences of stigma and discrimination (37). To illustrate how pervasive this is, in a series of studies, (9) monogamous targets were rated more positively than people in CNM relationships in relationship-relevant and relationship-irrelevant domains. That is, monogamous couples were perceived to not only be more trustworthy and passionate, but also to be more likely to pay their taxes on time, to floss their teeth, and to walk their dog (9). However, some forms of CNM, specifically polyamory, are viewed more favorably than other forms, such as swinging or open relationships $(26 ; 38)$. One reason that has been proposed for why people in CNM relationships continue to face stigma is because these relationships are associated with greater perceived promiscuity and likelihood of having sexually transmitted infections (STIs). In fact, research suggests that attitudes towards people in CNM relationships are related to the perception that people in CNM relationships will spread STIs (e.g., among polyamorists, see 39; and CNM more broadly, see 9). Indeed, in a study ( 38) examining stigma towards people in the differing types of CNM compared to monogamists showed that people in monogamous relationships are perceived to be the least promiscuous and to have the lowest STI rates while swingers were perceived as the most, and those in polyamorous and open relationships were in-between. Results also suggest that stigma towards people in CNM relationships was partially attributed to the perception of STI risk but not to perceptions of promiscuitysuggesting stigmatization towards people in CNM relationships, in part, was a function of perceptions of STI risk.

Notwithstanding these perceptions, people in CNM relationships are less likely to contract a sexually transmitted infection than people who identify as monogamous (see 40 for 
a review), and CNM individuals are more likely to engage in safer sex practices (e.g., using condoms and getting tested for STIs) than people in monogamous relationships (41-42). Part of the reason for these differences is that while people in CNM relationships might engage in sex with multiple partners, all partners are aware of the arrangement and can have an open discussion about their sexual health and extradyadic engagement. In the US, $20-25 \%$ of people in monogamous relationships engaged in extramarital sex (43-45) and people who are in monogamous relationships are less likely to practice safer sex in these encounters than CNM individuals $(40 ; 41 ; 46)$. Therefore, with infidelity occurring in a reliable minority of romantic relationships, apprehension about CNM relationships and concern about STI risk might be overblown while concern for STI risk among people in monogamous relationships may be understated. This idea is consistent with recent findings that suggest that monogamy might be less effective at preventing STIs than expected (see 40-41).

Stigma about CNM relationships also extends to the idea that opening up a relationship or having multiple romantic or sexual partners indicates that an existing or primary relationship is unsatisfying. However, a growing body of research suggests that people in CNM relationships are as equally satisfied with and committed to their relationships as individuals in monogamous relationships (15), and there is evidence that individuals in CNM relationships report higher sexual satisfaction and passion (47) and lower jealousy (48-49) than monogamists. Although there is growing evidence that people in CNM relationships report at least as much relationship quality on average as monogamous couples, CNM arrangements involve multiple partners and relationship and sexual outcomes across partner can differ. Recent research has found some differences between primary and secondary partners. For example, individuals in polyamorous relationships report being more satisfied 
with, committed to, and invested in their relationships with primary partners compared to secondary partners, but, they report higher sexual frequency $(17 ; 33)$, greater sexual satisfaction (47) and met sexual ideals (50) with their secondary partner compared to their primary partner. Research comparing reports for polyamorous partners to monogamous partners suggests that reports for primary partners in polyamorous relationships often mirror reports for monogamous partners. More specifically, a study comparing reports for polyamorous partners to monogamous partners found that reports for primary partners and monogamous partners do not differ with regards to commitment or investments, but people in polyamorous relationships tend to report higher sexual frequency, more stigma (e.g., less acceptance from friends and family) and stigma management (e.g., maintaining relationships in secrecy) with secondary partners compared to reports for monogamous partners (17).

\section{Broadening Our Understanding of Need Fulfillment}

One of the potential benefits of engaging in CNM relationships is the diversification of need fulfillment across multiple partners simultaneously. For example, in her book based on her experiences as a couples' therapist, Esther Perel (51) talks about how people's expectations for their romantic relationships have changed over time such that people now expect their romantic partners to meet several higher order needs, such as personal growth and emotional and sexual fulfillment. Perel's (51) ideas are in line with a model recently proposed by Finkel and colleagues (52) — the suffocation model of marriage - which argues that in Western culture, today relative to the past, people expect more from their relationships, and although people who can meet these high expectations can flourish, many people are not investing the time and energy to meet the high expectations they place on their relationships. The authors describe this as climbing mount Maslow without sufficient oxygen-meaning, people are 
aiming to meet higher order need for fulfillment and self-actualization without devoting the proper resources (52). In response to this model, Conley and Moors (53) proposed that adopting the tenets of CNM relationships and offloading some needs to additional partners could help strengthen or oxygenate a relationship — serving as one path toward greater need fulfillment.

Recent qualitative research with people in CNM relationships found that the most cited benefit of CNM reported by $42 \%$ of the sample) was diversified need fulfillment (54). In fact, in a study that followed people who were considering opening up an existing relationship found that one reason people seek out additional partners is to meet needs that are unfulfilled in their current relationships (55). In this study, people who actually opened up to a CNM relationship (compared to those who did not) reported greater sexual satisfaction over the course of two months, and this was especially true if they opened up their relationship to address sexual incompatibilities with their primary partner (55). Indeed, past research has shown that relationships with primary partners are characterized by more commitment, investments, and satisfaction and greater communication than relationships with secondary partners $(17 ; 33)$, and people tend to rate their primary partners as more desirable long-term partners (56). But, relationships with secondary partners are characterized by a greater percentage of time spent on sexual activity $(17 ; 33)$, more eroticism (18), more passion, and greater sexual satisfaction (47) and yet, secondary partners are considered less desirable longterm mates (56). Taken together this work provides preliminary evidence that primary and secondary relationships may meet different needs, with secondary relationships being characterized as more sexually fulfilling while primary relationships might meet more emotional needs. 
If individuals in CNM relationships can experience higher need fulfilment through having their needs met across multiple, simultaneous relationships, it is possible that the diversification of needs could be associated with feelings of satisfaction in their relationships. Mitchell and colleagues (19) proposed three different models to explain the role of need fulfillment in CNM relationships. The proposed models posit that having needs met in one relationship could either: (1) detract from the relationship satisfaction in another, concurrent relationship (e.g., contrast model), (2) be associated with greater satisfaction in another, concurrent relationship (e.g., additive model; 57), or (3) be associated with greater satisfaction in another relationship, but particularly when need fulfillment in the initial relationship is low (e.g., compensation model; 58). Several studies have examined the effects of need fulfillment in CNM relationships, but support for the different models has been mixed. For example, Mitchell and colleagues (19) found that the extent to which one partner met a person's needs was unrelated to satisfaction or commitment with another partner (inconsistent with the additive or contrast models) and need fulfillment across various needs assessed were consistently high with both partners (inconsistent with a compensation effect as people in CNM relationships were fulfilled by both partners). However, in a study (20) that investigated sexual need fulfillment specifically, when a primary partner was more motivated to meet a person's sexual needs, this was associated with greater sexual satisfaction with their secondary partner (evidence for the additive model), though greater sexual need fulfilment with a secondary partner was associated with less satisfaction with a primary partner (evidence for the contrast model). Similarly, a recent study examining sexual and emotional need fulfilment (18) found that when polyamorous individuals reported more eroticism (i.e., feelings of arousal, passion, lust, sexual pleasure) with their primary partners they reported greater sexual 
satisfaction with their secondary partner (evidence for the additive model) though greater eroticism with a secondary partner was associated with less sexual satisfaction with a primary partner (evidence for the contrast model). In contrast, ratings of one partner's nurturance (e.g., feelings of intimacy, warmth, and love) were not associate with sexual satisfaction in other, concurrent relationships suggesting that loving, caring feelings with one partner was not associated with sexual satisfaction with the other partner (18).

The findings on need fulfillment across relationships suggest that concurrent relationships can have null, negative, or positive effects on each other, and thus an important future direction will be understanding when, and under what circumstances, relationships with concurrent partners benefit or detract from relationship quality with the other. Although there is limited research to draw on at this time, it might be the case that certain qualities from a primary partner influence the ability to maximize the benefits of seeking out multiple, simultaneous relationships. For example, Muise and colleagues (20) found that when people in CNM relationships had a primary partner who was more communal (e.g., motivated to meet their needs), they were able to be more satisfied in their secondary relationships- suggesting that having a communal primary partner (or other features of the primary) can help people maximize the benefits of multiple concurrent relationships. It also might be the case that people can mitigate the detriments through their own actions. For example, Mogilski and colleagues (56) found that CNM participants reported talking about their extra-dyadic sexual experiences and downplaying these sexual experiences more often with their primary partner compared to their secondary partner, perhaps because sexual engagement is higher with this partner and thus restricting information may help individuals meet their needs while also maintaining their relationship with their primary partner. 


\section{Expanding Theories of Relationships Theoretical Advancements}

One of the unique benefits to studying CNM is that it informs and can challenge the boundaries of our current relationship theories. Indeed, scholars have recently argued that many theories in the psychology of relationships and sexuality include conceptualizations of relationship quality that have an implicit assumption that monogamy is the most desirable relationship style (47). As such, most of the theories used to understand and predict relationship processes have been tested with a monogamous sample and it is not clear how these might extend to sample of people who are navigating multiple relationships. There are two illustrative examples of this in the field of relationship and sexual science. First, one of the standard approaches for assessing love in relationships involves asking participants about their passionate and companionate love for their partner (59-60). However, this approach may inhibit researchers from understanding passionate and companionate love in polyamorous relationships. For example, the Passionate Love Scale (60) includes the item 'I'd get jealous if I thought [my partner] were falling in love with someone else." This item has a built in assumption that more jealousy about a third party is equivalent to more passionate love; however, researchers point out that this may not be true for individuals in CNM relationships who may actually experience positive affect in response to a partner finding a new relationship (i.e., people in polyamorous relationships may feel positive emotions when a loved one pursues other relationships sexually or emotionally; e.g., 48; 49; 61; 62). As a result of this assumption, participants who are in CNM relationships would score lower on passionate love due to lower levels of reported jealousy despite experiences of passion with partners (see 47). To address these issues, recent research has re-conceptualized the concepts of companionate and passionate love developing a measure that assesses eroticism and nurturance and that is 
applicable to both those in monogamous and CNM relationships (18).

Second, attachment theory is arguably among the most widely studied theories of romantic relationships. Attachment theory posits that romantic bonds are important sources of support, emotional stability, and safety and that secure attachment comes from repeated exposure to safe and supportive care (63). A small, but growing body of research, has found that individuals in CNM relationships report high relationship quality, open communication, high levels of honesty, trust, and intimacy, and low levels of jealousy (e.g., 17; 33; 48; 56;), all qualities that are analogous to those that characterize a secure attachment (65). However, one component that differs is relational exclusivity — or the assumption that pair bonding and love occurs among those who are sexually and romantically exclusive with their partners. In fact, sexual and romantic exclusivity are often conflated with ideas of love among attachment theorists (e.g., 66-68). Therefore, examining attachment among people in CNM relationships and across partners allows for novel theoretical testing of the bounds of attachment theory-and other theories_-given that CNM relationships afford people the opportunity to form deep emotional and sexual bonds with more than one person. Indeed, despite the theoretical assumptions (e.g., assuming pair bonds occur among exclusive, romantic dyads), recent work suggests that people in CNM relationships have similar attachment orientations with each of their partners (though greater security was reported for their primary partner) and report greater security (69) compared to established norms among monogamist (21). This work and other work (e.g., evidence for diversifying need fulfillment; 51-52), begs the question of the built-in assumptions in our theories while providing novel tests of the boundaries of existing theories.

\section{Conclusions and Future Directions}

The research on CNM to date suggests that a non-trivial number of people are 
interested in or have experience with CNM relationships, and that CNM relationships can be a healthy and viable alternative for those who are interested in relationships that extend beyond the monogamous dyad. Past work suggests that engaging in CNM can be associated with important benefits, such as the opportunity to diversify need fulfilment across partners, but also some challenges, such as enduring stigma and misunderstandings. Despite the burgeoning research in this area, many questions remain unanswered and are important future directions for researchers to pursue.

First, the bulk of the data on CNM relationships, as with monogamous relationships, includes people who are highly satisfied with their relationships. Therefore, we have little information about people who experience challenges with CNM or about people who have ended CNM relationship. Due the stigma around CNM relationships, an early goal of researchers seemed to be to compare CNM relationships to monogamous relationship to determine if engaging in CNM was, in fact, associated with more negative relationship and personal outcomes. Now that we have evidence that CNM relationships are at least as satisfying as monogamous relationships and can be a healthy, viable alternative for those who are interested, future research could explore more nuanced questions about the benefits and challenges of navigating relationships with multiple partners. Recently, a group of prominent CNM researchers have joined forces to develop a Multi-partner Relationship Maintenance Strategies Scale (MRMSS) that will assess how people who pursue multi-partner romance (e.g., polyamory, open relationships, swinging, plural marriage, etc.) regulate their own and their partners' sexual and intimate interactions with other people. The researchers seek to understand the practices that help people resolve or agitate common sources of conflict among romantic partners and rivals within multi-partner relationships (e.g., jealousy, partner rivalry, 
disease transmission, partner abandonment, etc.).

Additionally, the growing body of research on CNM has either utilized existing scales that have been created with the implicit assumption that monogamy is the most desirable relationship style (47) or has had to create their own scales to more adequately capture relational processes beyond the dyad (e.g., 18). This is a limitation of much of the existing literature, that aspects of existing theories of positive relationships and sexuality may not extend to people in CNM relationships. Moving forward it is important to think about whether our concepts and measures are inclusive to people in diverse relationships, including those in CNM relationships. This might mean stepping back and re-examining how we ask about commitment (do we assume commitment is equate with monogamy?) and how we ask about infidelity (do we assume any extradyadic relationships are cheating?) and other major constructs of interest. Therefore, there are opportunities for future research to expand our understanding of relationship quality and maintenance by re-considering the role of monogamy and by testing whether tenets of CNM relationships have broad implications for understanding need fulfilment.

\section{Acknowledgments}

This work has been supported by a Mitacs Accelerate and Elevate Grant awarded to Rhonda Balzarini and an Insight Development Grant from the Social Science and Humanities Research Council (SSHRC) awarded to Amy Muise and Rhonda Balzarini.

\section{Conflict of Interest}

Rhonda Balzarini declares the receipt of grants from Mitacs and the Social Sciences and Humanities Research Council during the preparation of this paper. 


\section{References}

1. United Nations (2015). World Population Ageing. Department of Economic and Social Affairs, Population Division. Retrieved from http://www.un.org/en/development/desa/population/publications/pdf/ageing/WPA2015_ Report.pdf

2. Rosenfeld, M. \& Byung-Soo, K. (2005). The independence of young adults and the rise of interracial and same sex unions. American Sociological Review 70, 541562.

3. Tamura, R., Murphy, K. M., \& Simon, C. J. (2008). Fertility decline, baby boom and economic growth. Journal of Human Capital 2, 262-302.

4. Tamura, R., \& Simon, C. (2016). Secular fertility declines, baby booms and economic growth: International evidence. Macroeconomic Dynamics, 21, 1601-1672.

5. Conley, T. D., Ziegler, A., Moors, A. C., Matsick, J, Valentine, B. (2013). A critical examination of popular assumptions about the benefits and outcomes of monogamous relationships. Personality and Social Psychology Review, 2, 124-141.

6. Ryan, C., \& Jetha, C. (2010). Sex at dawn: How we mate, why we stray, and what it means for modern relationships. New York, NY: Harper.

7. Bittman, M. \& Pixley, J. (1997). The double life of the family. Sydney, AU: Allen \& Unwin.

8. Stacey, J. (1996). In the name of the family: Rethinking family values in the postmodern age. Boston, MA: Beacon Press.

9. Conley, T. D., Moors, A. C., Matsick, J. L. \& Ziegler, A. (2013). The fewer the merrier? Assessing stigma surrounding consensually non-monogamous romantic relationships. 
Analyses of Social Issues and Public Policy, 13, 1-30.

10. Grunt-Mejer, K., \& Campbell, C. (2016). Around consensual nonmonogamies:

Assessing attitudes toward nonexclusive relationships. Journal of Sex Research, 53, 45-53.

11. Sizemore, K. M., \& Olmstead, S. B. (2017). A systematic review of research on attitudes towards and willingness to engage in consensual non-monogamy among emerging adults: Methodological issues considered. Psychology \& Sexuality, 8, 4-23.

12. Lebowitz, S. (2017). 10 things everyone wants to know about their relationship, according to Google. Business Insider. Retrieved from https://www.businessinsider.in/10-things- everyone-wants-to-know-about-theirrelationship-according-to- Google/articleshow/62058008.cms

13. Moors, A. C. (2016). Has the American public's interest in information related to relationships beyond "the couple" increased over time? Journal of Sex Research, 54, 677-684.

14. Khazan, O. (2016). OkCupid adds a feature for the polyamorous. The Atlantic. Retrieved from https://www.theatlantic.com/technology/archive/2016/01/ok-cupid-is-openingup-to- polyamorous-relationships/423162/

15. Rubel, A. N., \& Bogaert, A. F. (2015). Consensual nonmonogamy: Psychological wellbeing and relationship quality correlates. Journal of Sex Research, 52, 961-982.

16. Barker, M., \& Langdridge, D. (2010). Whatever happened to non-monogamies?

Critical reflections on recent research and theory. Sexualities, 13, 748-772.

17. Balzarini, R. N., Dharma, C., Kohut, T., Campbell, L., Lehmiller, J. J., Harman, J. J., \& Holmes, B. M. (2019). Comparing relationship quality across different 
types of romantic partners in polyamorous and monogamous relationships. Archives of Sexual Behavior, 48, 1749-1767.

- This article presents research demonstrating differences in reports of relationship quality for partners in polyamorous and monogamous relationships.

18. Balzarini, R. N., Dharma, C., Muise, A., \& Kohut, T. (2019). Eroticism versus nurturance: How eroticism and nurturance differ in polyamorous and monogamous relationships. Social Psychology, 50, 185-200.

19. Mitchell, M. E., Bartholomew, K. \& Cobb, R.C. (2014). Need fulfillment in polyamorous relationships. Journal of Sex Research, 51, 329-339.

20. Muise, A., Laughton, A., Moors, A. C., \& Impett, E. A. (2019). Sexual need fulfillment and satisfaction in consensually nonmonogamous relationships. Journal of Social and Personal Relationship, 36, 1917-1938.

21. Fraley, R. C. (2019). Attachment in adulthood: Recent developments, emerging debates, and future directions. Annual Review of Psychology, 70, 401-422.

22. Fairbrother, N., Hart, T. A., \& Fairbrother, M. (2019). Open relationship prevalence, characteristics, and correlates in a nationally representative sample of Canadian adults. The Journal of Sex Research, 56, 695-704.

23. Haupert, M., Gesselman, A., Moors, A., Fisher, H., \& Garcia, J. (2017). Prevalence of experiences with consensual non-monogamous relationships: Findings from two nationally representative samples of single Americans. Journal of Sex \& Marital Therapy, 43, 424-440.

24. Lehmiller, J. J. (2018). Tell me what you want: The science of sexual desire and how it can help you improve your sex life. Boston, MA: DaCapo. 
25. Barker, M. (2011). Monogamies and non-monogamies - A response to: 'The challenge of monogamy: Bringing it out of the closet and into the treatment room' by Marianne Brandon. Sexual and Relationship Therapy, 26, 281-287.

26. Matsick, J. L., Conley, T. D., Ziegler, A., Moors, A. C., \& Rubin, J. D. (2014). Love and sex: Polyamorous relationships are perceived more favourably than swinging and open relationships. Psychology and Sexuality, 5, 339-348.

27. Klesse, C. (2006). Polyamory and its 'others': Contesting the terms of non-monogamy. Sexualities, 9, 565-583.

28. Adam, B. D. (2006). Relationship innovation in male couples. Sexualities, 9, 5-26.

29. Jenks, R. J. (1998). Swinging: A review of literature. Archives of Sexual Behavior, 27, 507-521.

30. Easton, D., \& Hardy, J. W. (2009). The ethical slut (2nd ed.). Berkeley, CA: Ten Speed Press.

31. Taormino, T. (2008). Opening up. San Francisco, CA: Cleis Press.

32. Veaux, F. (2011). Care and feeding of polyamorous secondary relationships. More Than Two. Retrieved from https://www.morethantwo.com/primarysecondary.html

33. Balzarini, R. N., Campbell, L., Kohut, T., Holmes, B. M., Lehmiller, J. J., Harman, J. J., \& Atkins, N. (2017). Perceptions of primary and secondary relationships in polyamory. PLoS ONE, 12, e0177841.

- This article presents research demonstrating differences in reports of relationship quality across primary and secondary partners in polyamorous relationships.

34. Buchanan, D. R., Poppen, P. J., and Reisen, C. A. (1996). The nature of partner relationship 
and AIDS sexual risk-taking in gay men. Psychology and Health, 11, 541-555.

35. Poppen, P. J., Reisen, C. A., Zea, M. C., Bianchi, F. T., \& Echeverry, J. J. (2004). Predictors of unprotected anal intercourse among HIV-positive Latino gay and bisexual men. AIDS Behavior, 8, 379-389.

36. Moors, A. C., Matsick, J. L., Ziegler, A., Rubin, J. \& Conley, T. D. (2013). Stigma toward individuals engaged in consensual non-monogamy: Robust and worthy of additional research. Analyses of Social Issues and Public Policy, 13, 52-69.

37. Fleckenstein, J., Bergstrand, C.R., \& Cox, D.W. (2012). What Do Polys Want?: An Overview of the 2012 Loving More Survey. Loving More. Retrieved from http://www.lovemore.com/polyamory-articles/2012-lovingmore-polyamorysurvey/

38. Balzarini, R. N., Shumlich, E., Kohut, T., \& Campbell, L. (2018). Dimming the "halo" around monogamy: Re-assessing stigma surrounding consensually nonmonogamous romantic relationships as a function of personal relationship. Frontiers in Psychology, 894.

39. Johnson, S. M., Giuliano, T. A., Herselman, J. R., \& Hutzler, K. T. (2015). Development of a brief measure of attitudes towards polyamory. Psychology \& Sexuality, 6, 325-339.

40. Lehmiller, J. J. (2015). A comparison of sexual health history and practices among monogamous and consensually nonmonogamous sexual partners. Journal of Sexual Medicine, 12, 2022-2028.

41. Conley, Moors, Ziegker, \& Karathanasis, 2012; Conley, T. D., Moors, A. C., Ziegler, A., \& Karathanasis, C. (2012). Unfaithful individuals are less likely to practice 
safer sex than openly nonmonogamous individuals. Journal of Sexual Medicine, 9, 1559-1565.

42. Hutzler, K. T., Giuliano, T. A., Herselman, J. R., \& Johnson, S. M. (2016). Three’s a crowd: Public awareness and (mis)perceptions of polyamory. Psychology and Sexuality, 7, 69-87.

43. Greeley, A. (1994). Marital infidelity. Society, 31, 9-13.

44. Laumann, E. O., Gagnon, J. H., Michael, R. T., \& Michaels, S. (1994). The Social Organization of Sexuality: Sexual Practices in the United States. Chicago, IL: University of Chicago Press.

45. Wiederman, M. W. (1997). Extramarital sex: prevalence and correlates in a national survey. Journal of Sex Research, 34, 167-174.

46. Hinton-Dampf, A. M. (2011). Non-monogamous individuals compared to monogamous individuals: The differences in their relationships, specifically sexual risk behaviors and level of trust. Doctoral dissertation, University of Missouri, Kansas City, MO.

47. Conley, Matsick, Moors, \& Ziegler, 2017 Conley, T. D., Matsick., J., Moors., A. C., \& Ziegler, A. (2017). Investigation of consensually nonmonogamous relationships: Theories, methods, and new directions. Perspectives on Psychological Science, 12, 205-232.

48. Balzarini, R. N., McDonald. J., Kohut, T., Harman, J. J., Lehmiller, J. J., \& Holmes, B. M. (in press). Compersion: When jealousy inducing situations don't (just) induce jealousy. Archives of Sexual Behavior.

49. Mogilski, J. K., Reeve, S. D., Nicolas, S. C. A., et al. (2019). Jealousy, consent, and compersion within monogamous and consensually nonmonogamous romantic 
relationships. Archives of Sexual Behavior, 48, 1811-1828.

50. Balzarini, R. N., \& Muise, A. (conditional acceptance). Does diversifying sexual need fulfilment across partners in polyamorous relationships buffer the detriments of unmet sexual ideals? Archives of Sexual Behavior.

51. Perel, E. (2007). Mating in captivity: Unlocking erotic intelligence. New York, NY: HarperCollins.

52. Finkel, E. J., Hui, C. M., Carswell, K. L., \& Larson, G. M. (2014). The suffocation of marriage: Climbing Mount Maslow without enough oxygen. Psychological Inquiry, 25, 1-41.

53. Conley, T. D., \& Moors, A. C. (2014). More oxygen please! How polyamorous relationship strategies might oxygenate marriage. Psychological Inquiry, 25, 56-63.

54. Moors, A. C., Matsick, J. L., \& Schechinger, H. (2017). Unique and shared relationship benefits of consensually non-monogamous and monogamous relationships: A review and insights for moving forward. European Psychologist, 22, 55-71.

- This article reviews research CNM and monogamous relationships and examines the unique and shared benefits of engaging in either CNM or monogamous relationships, discussing implications and future directions of this research so far.

55. Murphy, A. P., Joel, S., \& Muise, A. (2020). A prospective investigation of the decision to open up a romantic relationship. Social Psychological and Personality Science, advanced online publication.

56. Mogilski, J. K., Memering, S. L., Welling, L. L., \& Shackelford, T. K. (2017). Monogamy versus consensual non-monogamy: Alternative approaches to pursuing a strategically pluralistic mating strategy. Archives of Sexual Behavior, 46, 407-417. 
57. Cook, E. (2005). Commitment in polyamorous relationships (Unpublished master's thesis). Regis University, Colorado.

58. Sheff, E. (2011). Polyamorous families, same-sex marriage, and the slippery slope. Journal of Contemporary Ethnography, 40, 487-520.

59. Hatfield, E., \& Rapson, R. L. (2013). Companionate love scale. Measurement Instrument Database for Social Science. Retrieved from www.midss.ie.

60. Hatfield, E., \& Sprecher, S. (1986). Measuring passionate love in intimate relationships. Journal of Adolescence, 9, 383-410.

61. Ritchie, A., \& Barker, M. (2007). Hot bi babes and feminist families: Polyamorous women speak out. Lesbian and Gay Psychology Review, 8, 141-151.

62. Sheff, E. (2014). The polyamorists next door: Inside multiple partner relationships and families. Lanham, MD: Rowman \& Littlefield.

63. Hazan, C., \& Shaver, P. R. (1994). Attachment theory as an organizational framework for research on close relationships. Psychological Inquiry, 5, 1-22.

64. Feeney, J. A. (2008). Adult romantic attachment: Developments in the study of couple relationships. In Cassidy, J., Shaver, P. R. (Eds.), Handbook of attachment: Theory, research, and clinical applications (2nd ed., pp. 456-481). New York, NY: Guilford Press.

65. Hazan, C., \& Shaver, P. (1987). Conceptualizing romantic love as an attachment process. Journal of Personality and Social Psychology, 52, 511-524.

66. DeWall, C. N., Lambert, N. M., Slotter, E. B., Pond, R. S., Deckman, T., Finkel, E. J....Fincham, F. D. (2011). So far away from one's partner, yet so close to romantic alternatives: Avoidant attachment, interest in alternatives, and infidelity. Journal of 
Personality and Social Psychology, 101, 1302-1316.

67. Hazan, C., Campa, M., Gur-Yaish, N. (2006). What is adult attachment? In Mikulincer, M., Goodman, G. S. (Eds.), Dynamics of romantic love: Attachment, caregiving, and sex (pp. 47-70). New York, NY: Guilford Press.

68. Hazan, C., \& Zeifman, D. (1999). Pair bonds as attachments: Evaluating the evidence. In J. Cassidy \& P. Shaver (Eds.), Handbook of attachment (pp. 336-354). New York: Guilford Press.

69. Moors, A. C., Ryan, W., \& Chopik, W. J. (2019). Multiple loves: The effects of attachment with multiple concurrent romantic partners on relational functioning. Personality and Individual Differences, 147, 102-110. 